\title{
Hydrodynamic drag forces on two porous spheres moving along their centerline
}

\author{
R.M. Wu ${ }^{\mathrm{a}}$, H.Y. Chung ${ }^{\mathrm{b}}$, D.J. Lee ${ }^{\mathrm{b}, *}$ \\ ${ }^{a}$ Department of Chemical and Materials Engineering, Tamkang University, Tamsui, Taipei County 251, Taiwan \\ ${ }^{\mathrm{b}}$ Department of Chemical Engineering, National Taiwan University, Taipei 10617, Taiwan
}

Received 16 May 2003; received in revised form 8 November 2003; accepted 9 December 2003

\begin{abstract}
This paper numerically evaluates the hydrodynamic drag force exerted on two highly porous spheres moving steadily along their centerline (sphere \#1 and sphere \#2) through a quiescent Newtonian fluid over a Reynolds number ranging from 0.1 to 40 . At creeping flow limit, the drag forces exerted on both spheres were identical. At higher Reynolds numbers the drag force on sphere $\# 1$ was higher than sphere $\# 2$, revealing the shading effects produced by sphere $\# 1$ on sphere $\# 2$. At dimensionless diameter $\left(\beta,=d_{f} / 2 k^{0.5}, d_{f}\right.$ and $k$ are floc diameter and interior permeability, respectively) $>20$, the spheres can be regarded nonporous. At $\beta<20$, the drag forces dropped. At $\beta<2$, the drag forces approached "no-spheres" limit. An increased size ratio of two spheres $\left(d_{f 1} / d_{f 2}\right)$ would increase the drag force on sphere \#1 and reduce that on sphere \#2. At increasing $\beta$ for both spheres, the drag force on sphere \#2 was increased because of the more difficult advective flow through its interior, and at the same time the drag was reduced owing to the stronger wake flow produced by the denser sphere \#1. The competition between these two effects leads to complicated dependence of drag force on sphere $\# 2$ on $\beta$ value. These effects were minimal when $\beta$ became low. Two identical spheres could move steadily along their centerline. At higher Reynolds number, the two spheres would move closer because of the incorporation of inertia force. For spheres of different diameters, the sphere \# 2 would move faster than sphere \#1 regardless of their size ratio and $\beta$ value. This occurrence yielded efficient coagulation when two porous spheres were moving in-line.
\end{abstract}

(C) 2003 Elsevier Ltd. All rights reserved.

Keywords: Porous; Drag force; Centerline; Simulation

\section{Introduction}

Information on the hydrodynamic drag force exerted on a highly porous object is necessary when considering its motion, such as sedimentation or centrifugation of sludge flocs. Considering a porous sphere moving steadily through a quiescent, infinitely large liquid pool, the drag force exerted on the porous sphere can be stated as follows:

$F_{D}=\left(\frac{\pi}{4} d_{f}^{2}\right)\left(\frac{1}{2} \rho V^{2}\right) C_{D} \Omega$,

where $C_{D}$ is the drag coefficient, $d_{f}$ the sphere's diameter, $V$ the velocity, $\rho$ the fluid density, and $\Omega$ the ratio of the resistance experienced by a porous sphere to that by an equivalent solid sphere. The drag coefficient is a function of Reynolds

\footnotetext{
* Corresponding author. Tel.: +886-2-2363-5230; fax: +886-2-2362-3040.

E-mail address: djlee@ccms.ntu.edu.tw (D.J. Lee).
}

number $(R e)$ and floc sphericity (Clift et al., 1978; Tambo and Watanabe, 1979; Namer and Ganczarczyk, 1993). Under creeping-flow condition $(R e \ll 1), C_{D}$ for a nonporous sphere is governed by the Stokes' law, that is, $C_{D}=24 / R e$, $\Omega=1$, and $F_{S}=3 \pi \mu d_{f} V$. For a highly porous sphere moving steadily through an infinite medium, the corresponding drag force $\left(F_{D}\right)$ is less than that for a nonporous sphere $\left(F_{S}\right)$. Restated, $F_{D}=F_{S} \Omega$ and $\Omega<1$ (Chung et al., 2003). Neale et al. (1973) analytically derived the correction factor $\Omega$ for a moving porous sphere under creeping-flow condition. Matsumoto and Suganuma (1977) experimentally verified the theoretical predictions of Neale et al.

Lee et al. (1996) revealed that in many processes involving sludge flocs, the corresponding sphere's $R e$ could be up to 40 , at which the Stokes' law fails. However, previous literature has largely adopted the Stokes law-like correlation (i.e., $C_{D}=A / R e$, where $A$ is a constant) for satisfactorily interpreting the floc-settling data beyond the creeping-flow regime (Tambo and Watanabe, 1979; Mitani et al., 1983; 
Lee and Hsu, 1994; Hung et al., 1996; Chu et al., 1997). Our recent works demonstrated that, owing to the highly porous interior through which the advection flow can easily pass, the streamline would not be seriously distorted in the after-sphere regime (Tsou et al., 2002; Wu et al., 2002a). This occurrence has largely extended the Stokes law-like correlation beyond the creeping-flow regime (Wu and Lee, 1998a,b, 1999, 2001). In these works the floc was modeled as a highly porous sphere or nonspherical object of permeability $k$. With the free-settling tests measuring the size and the settling velocity of individual floc, the fractal dimension describing the global structure of floc interior could be obtained (Wu et al., 2002b).

Field applications normally incorporate more than one porous sphere, for example, millions of flocs settle together in the settling zone in the clarifiers. Interactions among particles are essential to affect their motion when the suspension could not be regarded as "diluted". Following the pioneer work of Stimson and Jeffery in 1926 there is a vast body of literature being devoted to the hydrodynamic interactions between two nonporous spheres moving slowly along the line of their centers (Happel and Brenner, 1983). If the two spheres were identical, then the hydrodynamic forces exerted on both spheres would be the same under creeping flow condition. Moreover, when two spheres are approaching each other, these two spheres form a doublet and could move faster than the single sphere under the same external force field. The drag forces exerted on the two identical spheres are defined as follows:

$F_{S}=3 \pi \mu d_{f} V \gamma$,

where $\gamma$ denotes the ratio between the drag force exerted on either sphere and the Stokes' law. Then, as $S / d_{f} \rightarrow$ $\infty$, where $S$ is the center-to-center distance, $\gamma \rightarrow 1$, a self-evident result. As $S / d_{f} \rightarrow 1.0$, that is, when two spheres touch, $\gamma \rightarrow 0.645$. It is clear from Eqs. (1) and (2) that $\gamma=\Omega$ if $R e<0.1$ and $\beta \rightarrow \infty$, where $\beta=d_{f} / 2 k^{0.5}$.

Works considering the interactive particles at intermediate to high Reynolds number include the use of some measuring apparatus to calibrate the exerted force on suspended particles (Lee, 1979; Tsuji et al., 1982, 1985; Zhu et al., 1994; Liang et al., 1996; Chen and Lu, 1998; Chen and Wu, 2000). For instance, Lee (1979) and Tsuji et al. (1982) measured the drag forces on the two-sphere system at $R e=10,000$ and $200-1000$, respectively. Their reported drag force is higher than that by Chen and $\mathrm{Wu}(2000)$, who measured the drag force and flow field between two spheres at Reynolds number ranging from 54 to 118 . Chen and $\mathrm{Lu}$ (1998) noted a reduction in drag force for the two interacting spheres at high Reynolds number, a similar trend also observed at creeping flow limit.

Despite of the extensive researches on the interactions between two moving spheres, few works analyzed the interactions between two highly porous spheres moving along their centerline at intermediate Reynolds number, which is commonly observed for processes including biological aggre- gates. We numerically investigated the fluid flow field within and around two porous spheres moving along their centerline at $R e=0.1$ or 40 . The corresponding hydrodynamic drag forces exerted on the spheres' surfaces were evaluated. Effects of sphere permeability and the center-to-center distance on the hydrodynamic drag force were discussed. Implications to field application were commented.

\section{Equations and solution}

We consider herein two spheres (\#1 and \#2), porous or nonporous, of diameters $d_{f 1}$ and $d_{f 2}$ and permeability $k_{1}$ and $k_{2}$, respectively, moving along their centerline at a steady speed of $V$ through an unbound quiescent Newtonian fluid of viscosity $\mu$ and density $\rho$. The center-to-center distance between the two spheres is termed $S$. For the sake of easy definition of boundary conditions in the computational domain, an equivalent problem is considered: the spheres are fixed at the centerline while the surrounding fluid is flowing at a uniform speed of $V$ from infinity towards the fixed spheres. Fig. 1 depicts schematically the flow process.

The governing equation for the fluid velocity $\vec{u}_{p}$ within the porous spheres taking into account the viscous effect is the so-called combined Darcy-Brinkman law, which is stated as follows:

$\vec{u}_{p}^{*}+E u R e \frac{1}{\beta^{2}} \nabla P^{*}=\frac{1}{\beta^{2}} \nabla^{2} \vec{u}_{p}^{*}$,

where $E u=P_{0} / \rho V^{2}$ (Euler number), $R e=R \rho V / \mu$ (Reynolds number), $\beta=d_{f} / 2 k^{0.5}, P^{*}=P / P_{0}$ and $\vec{u}_{p}^{*}=\vec{u}_{p} / V$. In Eq. (3), the interior fluid viscosity is assumed the same as that for the surrounding fluid (Neale et al., 1973). The two terms in the left-hand side of Eq. (3) is the Darcy's law, which is strictly valid for a porous sphere at a porosity of less than 0.5 . The right-hand side is attributed to the Brinkman law, a modification version of Darcy's law considering a highly porous medium. We have neglected inertia effect within the

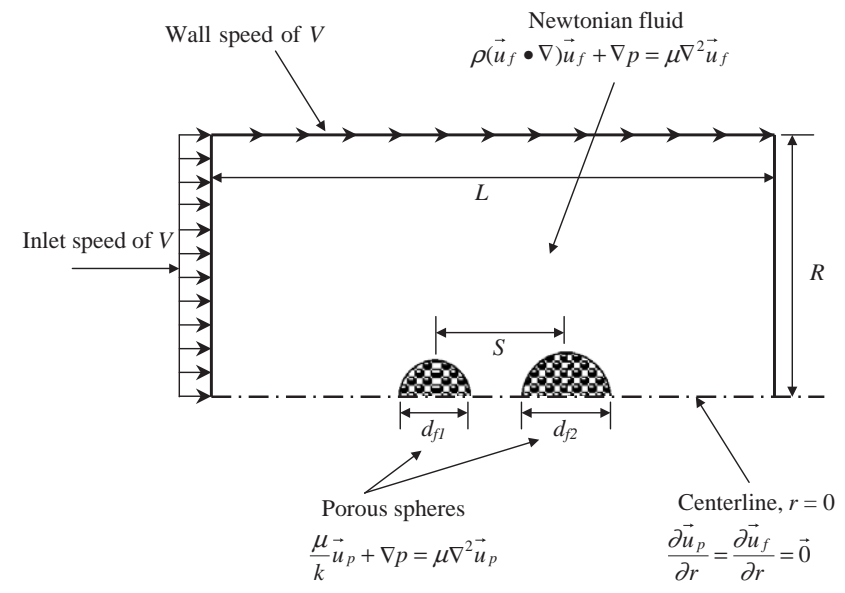

Fig. 1. Computation domain under investigation. 
porous sphere, which limits the applicable Reynolds number to be less than 40 .

For the surrounding Newtonian fluid field, the governing equations are the steady-state Navier-Stokes equations, which can be stated as follows:

$\left(\vec{u}_{f}^{*} \cdot \nabla\right) \vec{u}_{f}^{*}+\frac{P_{0}}{\rho V^{2}} \nabla P^{*}=\frac{1}{R e} \nabla^{2} \vec{u}_{f}^{*}$,

where $\vec{u}_{f}$ is the fluid velocity. The first and the second terms of the left-hand side of Eq. (4) correspond to the inertial and pressure effect, respectively; while the right-hand side, the viscous effect. Notably, the incorporation of the inertia term in Eq. (4) leads to nonlinear governing equations that require numerical solution.

The boundary conditions are as follows:

$\vec{u}_{f}=\vec{V} \quad$ at $r \rightarrow \infty$,

$\frac{\partial \vec{u}_{p}}{\partial r}=\frac{\partial \vec{u}_{f}}{\partial r}=\overrightarrow{0} \quad$ at $r=0$,

$\vec{u}_{p}=\vec{u}_{f} \quad$ at spheres' surface,

$\nabla \vec{u}_{p}=\nabla \vec{u}_{f} \quad$ at spheres' surface.

Eq. (5a) states that the surrounding fluid is moving at a constant speed when away from the sphere. Eq. (5b) addresses the symmetric line. Eqs. (5c) and (5d) are the continuation conditions of fluid velocity and shear stress across the sphere surface. For nonporous sphere case, Eq. (5c) was changed into $\vec{u}_{f}=\overrightarrow{0}$ to fulfill the no-slip boundary condition; while Eq. ( $5 d)$ is not used in calculation. We have more than single length scale in this system. The Reynolds number used in this work is based on the diameter of sphere of greater size, that is, $R e=\rho V d_{f 2} / \mu$ when $d_{f 1}<d_{f 2}$, and vice versa.

The computational fluid dynamics program FIDAP 7.6 (FDI Inc., USA) solved the governing equations, Eqs. (3) and (4), together with the associated boundary conditions Eqs. (5a)-(5d), using bilinear, four node quadrilateral elements. Three-dimensional simulation was conducted to show that the flow pattern was asymmetrical at a maximum Reynolds number of 40, hence, the axisymmetry-flow option is employed to reduce the original three-dimensional problem into a simpler, two-dimensional problem. The numerical solution cannot be accomplished in an infinite domain, as addressed in Eq. (5a). In numerical practice, the ratio $R / d_{f}$ would not influence the calculation results if they exceed eight. The domain tube can be used to represent an infinite flow field when the $R / d_{f}$ is fixed at 15 , twice as that to be affected by the existence of the spheres. It is noticeable that the wake could more effectively affect the downstream region than the region by the spheres' side. The $L / d_{f}$ ratio is set at 75 in the present work. The numbers of elements in the fluid side and within the porous sphere are about 14,000 and 60, respectively. Notably, since it is the fluid field within and around the porous sphere of major concern, more elements are allocated near the sphere for better accuracy. The calculations were carried out with maximum relative error of $10^{-3}$ in fluid velocity evaluation.
FIDAP evaluates the hydrodynamic drag force on the basis of the fluid velocity field within and around the porous sphere with its post-processing program FIPOST (FIDAP, 1993). The maximum relative errors for drag force evaluation are less than 3\% when compared with literature results (Bird et al., 2002). We herein define the $\Omega$ value as the ratio of force on a porous sphere moving together with other sphere(s) to a single, equivalent solid sphere moving at the same velocity in an unbound liquid.

\section{Results and discussion}

\subsection{Velocity field}

Fig. 2 demonstrates the velocity field plots for two identical spheres $\left(d_{f 1}=d_{f 2}\right)$ separated by a distance of $d_{f 2}\left(S / d_{f 2}=\right.$ 2 ), where $R e=0.1$ or 40 , and $\beta=500$ (nonporous spheres), 5 (commonly noted for sludge flocs), or 0.05 (approaching the "no-sphere" limit), respectively. For nonporous spheres, the originally flat-shaped flow field is markedly distorted when it approaches the spheres, owing to the no-slip boundary condition at the solid surface. At $R e=0.1$, the fluid moves mostly along (parallel to) the sphere surface (Fig. 2e), and hence the streamlines are nearly symmetrical in the front and the rear sides of the two sphere. The fluid flowing parallel to the solid surface is the basic characteristics of a creeping flow pattern, where only viscous force dominates (Denn, 1980).

At $R e=40$, on the other hand, since the pressure in the growing boundary layer from the leading stagnant point of sphere \#1 is less than that in the free stream, the deceleration of fluid will induce an adverse pressure gradient. The fluid tends to flow departed from the rear surface of sphere $\# 1$. (Although it is not very obvious, as Fig. $2 \mathrm{f}$ reveals, the flow has separated over the rear of the sphere, to form a thin standing vortex ring.) An essential flow pattern difference thereby occurs when compared with Fig. 2e. Since the fluid

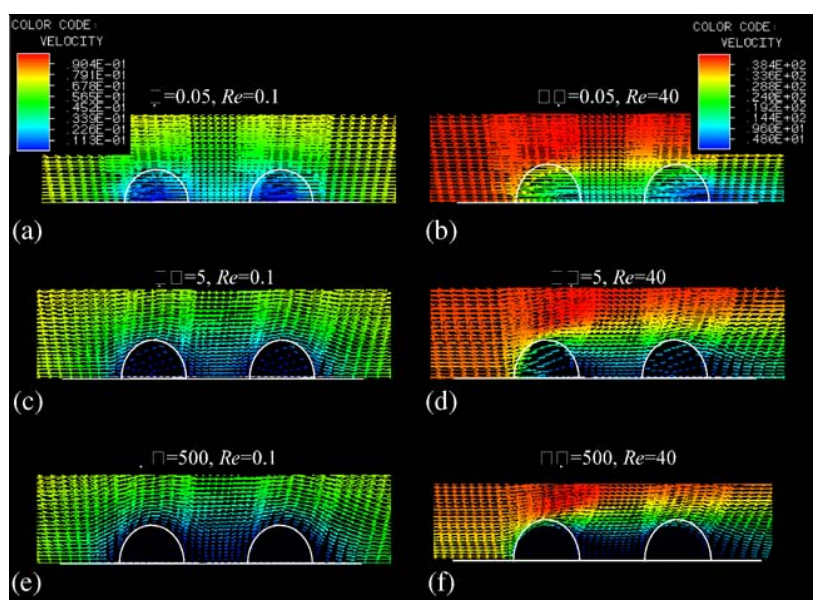

Fig. 2. The velocity fields for two identical spheres $\left(d_{f 1}=d_{f 2}\right) \cdot S / d_{f 2}=2.0$. 


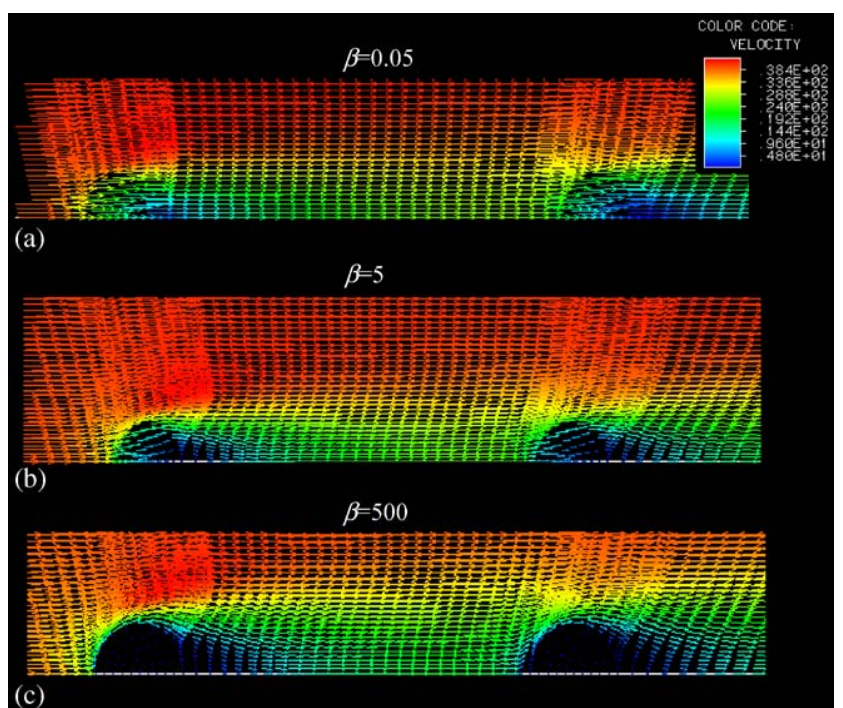

Fig. 3. The velocity fields for the two identical spheres at $S / d_{f 2}=5$.

does not flow parallel to the solid surface, inertia force has come into play, and, together with the viscous force, dominate the flow process.

For highly porous spheres, nevertheless, the fluid can not only flow around, but also flow through the porous sphere. At $R e=0.1$, the streamlines remain almost flat-shaped when passing over the porous spheres (Fig. 2a). Except along the central line of the sphere interior there is a sensible decrease in fluid velocity, the fluid velocity remains almost uninfluenced by the existence of the porous spheres. A similar pattern is noted at a Reynolds number of 40 (Fig. 2b), except that the corresponding velocity has become higher. The comparison between the fluid field of nonporous and porous spheres makes clear that the boundary layer separation and wake formation, which are mainly responsible for the departure of Stokes' law at elevated Reynolds numbers, have not occurred for highly porous spheres even at a Reynolds number of 40 . This occurrence corresponds to our previous speculations that owing to the easy advection flow through the floc interior, the boundary layer separation and the after-sphere wakes might not occur for highly porous spheres even moving at an intermediate Reynolds number. $\mathrm{Wu}$ and Lee (1998a) also noted no basic flow pattern difference exists for a single highly porous sphere moving at a Reynolds number of 0.1 or 40 .

Fig. 3 depicts the velocity fields for the two identical spheres at $S / d_{f 2}=5$. Apparently the interactions between the two spheres are weaker than those depicted in Fig. 2. At a very large $S$, the two spheres should move as a single sphere. When $d_{f 1}>d_{f 2}$, the corresponding $S$ value to neglect the mutual interactions between the spheres is longer; when $d_{f 1}<d_{f 2}$, the corresponding critical $S$ value is shorter.

\subsection{Drag force}

The drag force exerted on the two spheres can be numerically evaluated. Fig. 4 a depicts the numerical results at
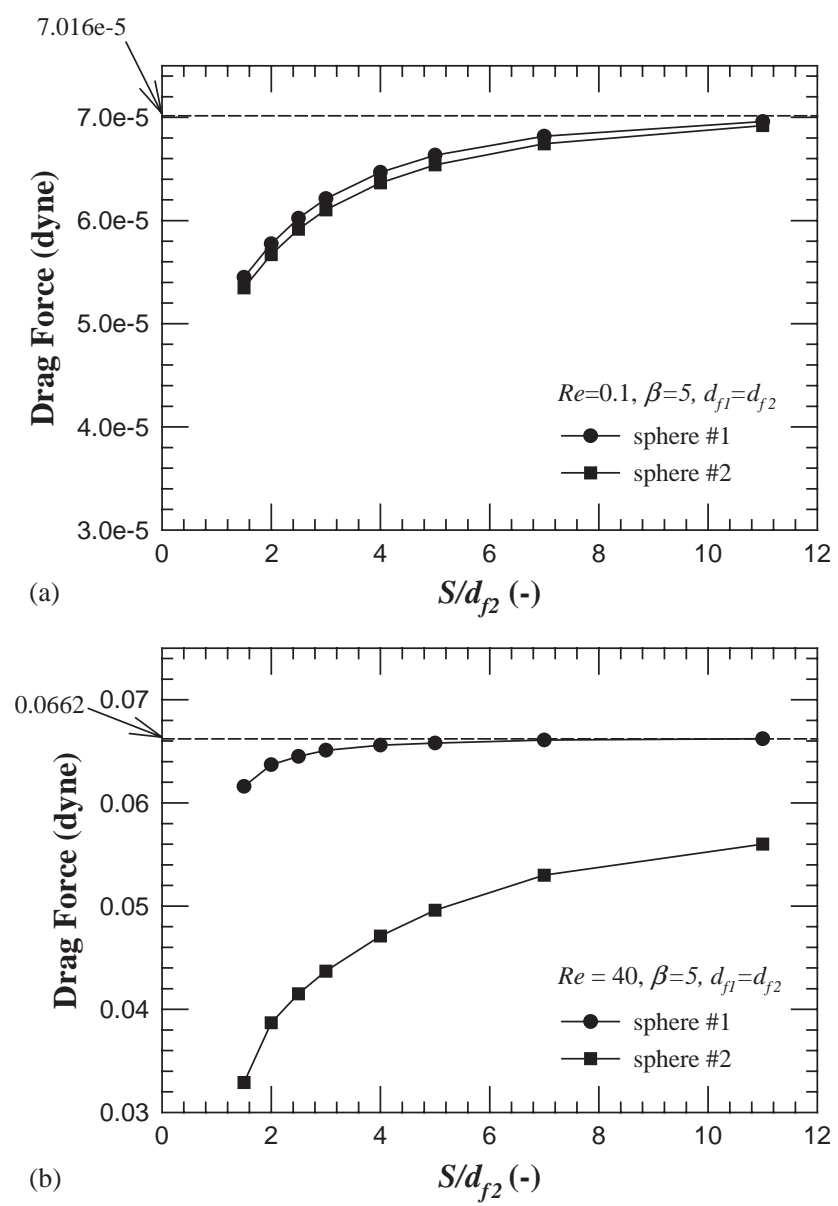

Fig. 4. The drag force exerted on the two identical spheres, $\beta=5$. (a) $R e=0.1$; (b) $R e=40$.

$d_{f 1}=d_{f 2}, \operatorname{Re}=0.1$, and $\beta=5$. The dashed lines indicate the drag forces for a single sphere of diameter $d_{f 2}$ moving at the same $R e$ and $\beta$ values. As Fig. 4a depicts, the drag force at $R e=0.1$ decreases with decreasing $\mathrm{S}$. For instance, at $S=11 d_{f 2}, F_{D}=6.92-6.96 \times 10^{-5}$ dynes; while at $S=1.5 d_{f 2}$, $F_{D}=5.35-5.45 \times 10^{-5}$ dynes. When the two spheres are separated apart by more than $10 d_{f 2}$, the drag force is little affected by $S$. At creeping flow limit, owing to the symmetrical flow patterns noted in Fig. 2e, the drag forces exerted on both spheres should be identical (Stimson and Jeffery, 1926). The discrepancy observed for drag forces on spheres $\# 1$ and \#2 (Fig. 4a) represents the numerical uncertainty, giving a relative error for drag force less than $2 \%$. In general the maximum relative errors in drag force evaluation are $3 \%$.

At $R e=40, F_{D 1}>F_{D 2}$ (Fig. 4b). The drag force on sphere \#1 approaches a plateau value at about $S=4 d_{f 2}$, much smaller than that for sphere $\# 1$ at $R e=0.1\left(\mathrm{ca} .10 d_{f 2}\right)$. Meanwhile, the drag force on sphere $\# 2$ still gradually increases at $S=10 d_{f 2}$. Hence, the incorporation of inertia force weakens the effects of sphere $\# 2$ on sphere $\# 1$, but enhances the effects of sphere \#1 on sphere \#2. 


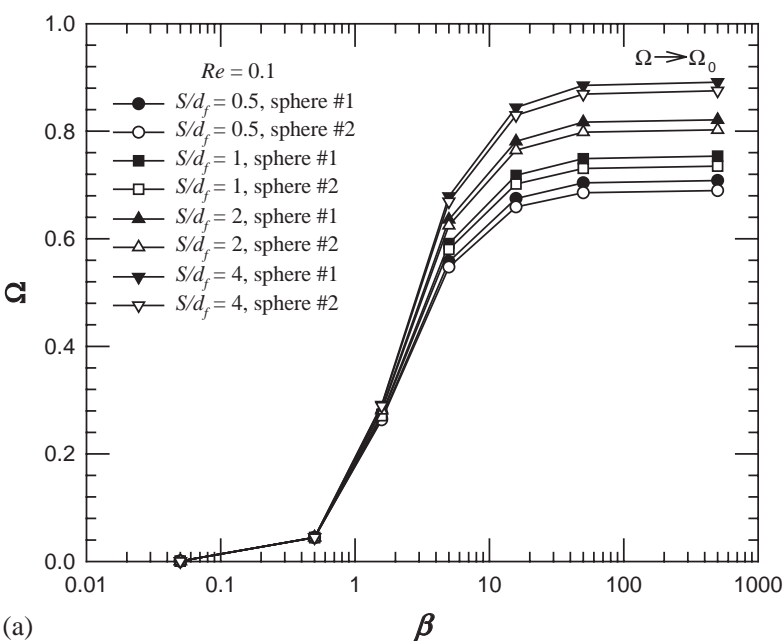

(a)

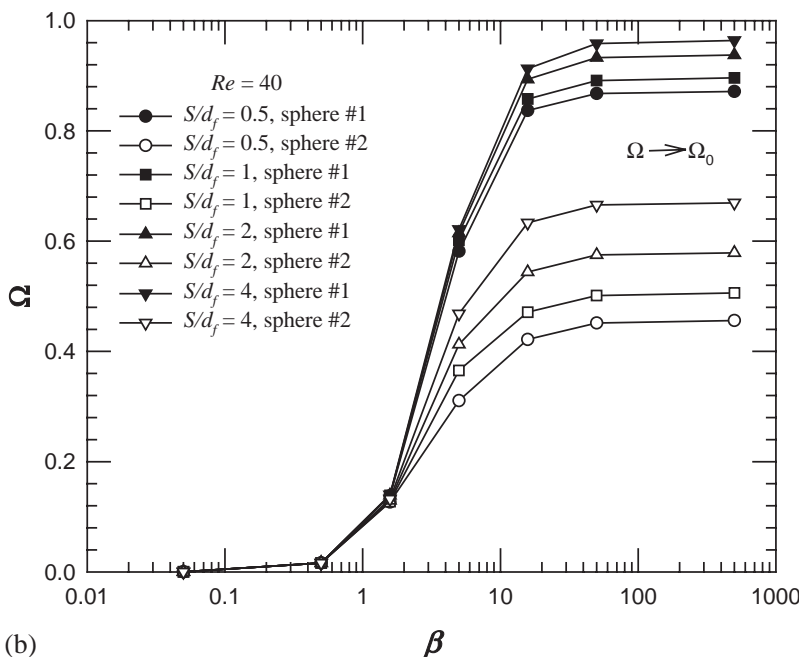

Fig. 5. Effects of $\beta$ value on the ratio of drag force exerted on the sphere (\#1 or \#2) to the corresponding drag force exerted on a single, nonporous sphere of size $d_{f 2} . d_{f 1}=d_{f 2}$ (a) $R e=0.1$; (b) $R e=40$.

Fig. 5 shows the implications of $\beta$ value on the ratio of drag force exerted on the sphere (\#1 or \#2) to the corresponding drag force exerted on a single, nonporous sphere of size $d_{f 2}(\beta \rightarrow \infty)$ under identical hydrodynamic environment $\left(d f=d_{f 1}=d_{f 2}, R e=0.1\right.$ or 40$)$ and with separation $S$ as a parameter. As the dimensionless floc permeability $\beta$ exceeds approximately 20 , the ratio is unity, denoting that the spheres can be regarded nonporous. At $\beta<20$, the corresponding ratio decreases from unity. For example, for both spheres at $R e=0.1$ and $S / d_{f 2}=2, \Omega=0.58$ at $\beta=15.8$, and $\Omega=0.27$ at $\beta=1.58$. While at $R e=40$, the corresponding $\Omega$ 's are 0.86 and 0.13 for sphere $\# 1$, and 0.46 and 0.125 for sphere $\# 2$, respectively. At an even lower $\beta$ value, the $\Omega$ ratio levels off and, at $\beta=0$ limit, approaches zero, denoting an extreme of "no-spheres". The complete ratio versus $\beta$-value curve is sigmoid-like, with the nonporous spheres ( $\left.\Omega=\Omega_{0}\right)$ and the "no-spheres" $(\Omega=0)$ conditions presenting the two extremes. In addition, the $\Omega$ value is strongly

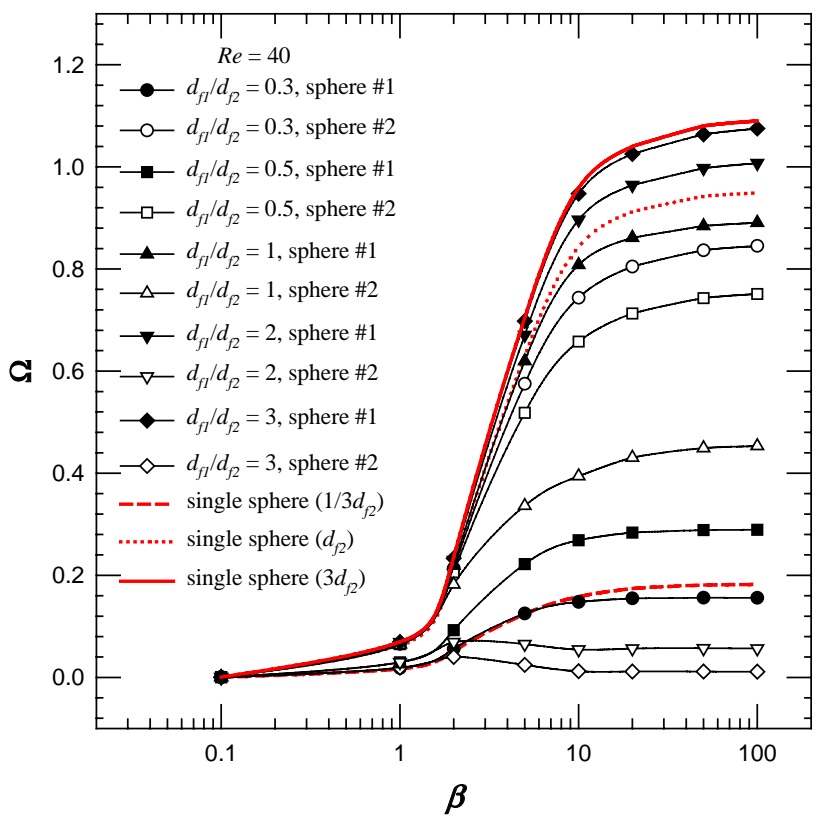

Fig. 6. Effects of $\beta$ value on the drag forces exerted on the sphere \#1 and sphere \#2. $R e=40$ and $S=d_{f 2}+\left(d_{f 1}+d_{f 2}\right) / 2$.

affected by the spheres' separation at $\beta>1.5$. This is the range for typical sludge flocs observed in previous studies (Wu et al., 1998, 2000a,b).

Fig. 6 shows the implications of $\beta$ value on the drag forces exerted on the spheres \#1 and \#2 at $R e=40$ and $S=d_{f 2}+\left(d_{f 1}+d_{f 2}\right) / 2$, with $d_{f 1} / d_{f 2}$ as a parameter. The force increases with the sphere's size, a self-evident result. At $\beta<0.1$, the $\Omega$ value is very low, indicating an easy advection flow through the sphere. As $\beta>20$, the spheres of different diameters could be regarded nonporous. An increased size ratio of $d_{f 1} / d_{f 2}$ would increase $F_{D 1}$ and reduce the $F_{D 2}$. A greater drag force exerted on the spheres when their interior becomes less porous, as commonly expected. Exception occurs for sphere \#2 at $\left(d_{f 1} / d_{f 2}\right)>1$, whose drag force would first increase with $\beta$ up to around 2, then drop and reach a local minimum at around $\beta=10$. This phenomenon is obviously attributable to the mutual interaction between sphere $\# 1$ and sphere $\# 2$, but had yet been satisfactorily addressed in pertinent literature.

To further explore the mechanisms corresponding to the noted hydrodynamic interactions demonstrated above, the stress distributions contributed by the skin drag or by the form drag are discussed in the next section.

\subsection{Form and skin drags}

The local normal stress and shear stress on sphere's surface are $\left(-\left.p\right|_{r=d_{f} / 2}\right) \cos \theta$ and $\left(\left.\tau_{r \theta}\right|_{r=d_{f} / 2}\right) \sin \theta$, respectively. The normal stress and the skin stress are evaluated 


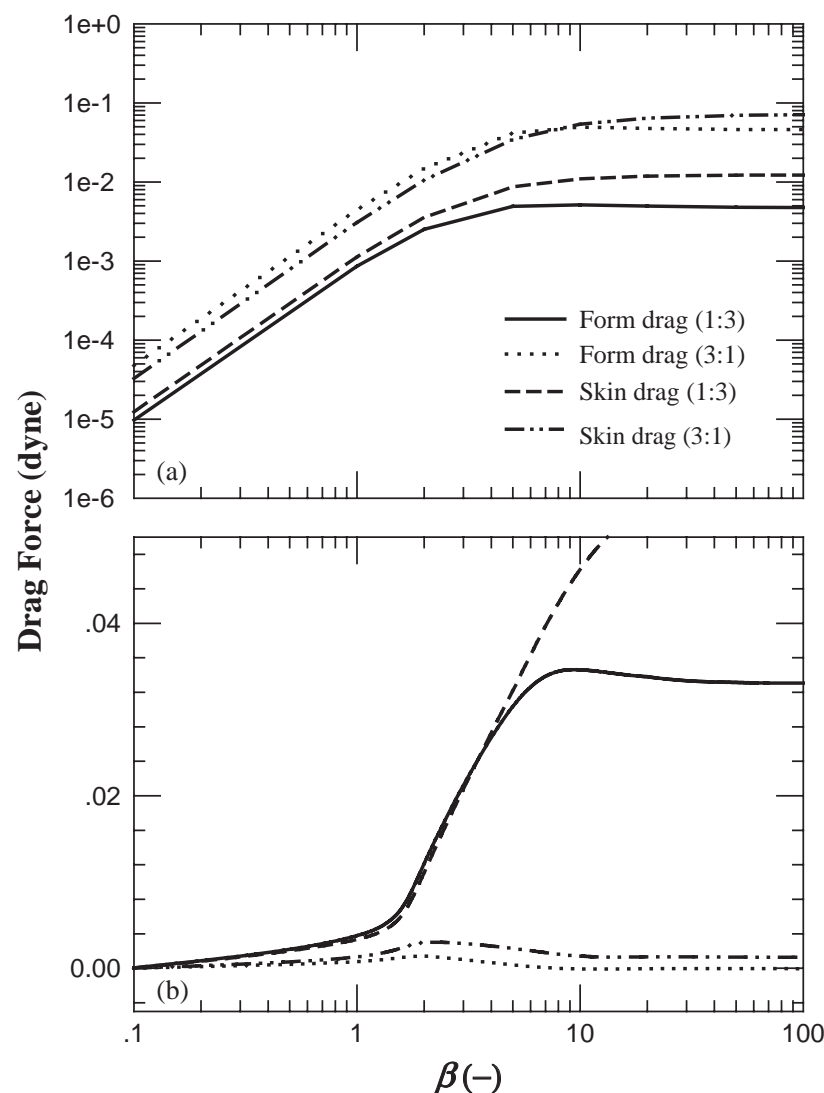

Fig. 7. The form drag and skin drag for the sphere \#1 and sphere \#2, $R e=40$. (a) Sphere \#1; (b) sphere \#2.

as follows:

$$
\begin{aligned}
& F_{\text {form }}=\int_{0}^{2 \pi} \int_{0}^{\pi}\left(\operatorname{Abs}\left(-\left.p\right|_{r=d_{f} / s}\right) \cos \theta\right) R^{2} \sin \theta \mathrm{d} \theta \mathrm{d} \phi, \\
& F_{\text {skin }}=\int_{0}^{2 \pi} \int_{0}^{\pi}\left(\left.\tau_{r \theta}\right|_{r=d_{f} / 2} \sin \theta\right) R^{2} \sin \theta \mathrm{d} \theta \mathrm{d} \phi .
\end{aligned}
$$

The total drag force is the sum of $\left(F_{\text {form }}+F_{\text {skin }}\right)$.

Figs. $7 \mathrm{a}$ and $\mathrm{b}$ show the form drag and skin drag for the sphere $\# 1$ and sphere $\# 2$, respectively, at $R e=40$ and at $d_{f 1} / d_{f 2}=1 / 3$ or $3 / 1$. For sphere $\# 1$, the form drag becomes more profound than the skin drag at increasing $d_{f 1} / d_{f 2}$. The larger the sphere, the more significant is the role of form drag, owing to the greater streamline distortion over the sphere. At $\beta<10$ for $d_{f 1} / d_{f 2}=3 / 1$ or $\beta<2$ for $d_{f 1} / d_{f 2}=$ $1 / 3$, both drags increase with $\beta^{2}$, indicating the "highly porous" limit. $\operatorname{Atd}_{f 1} / d_{f 2}=3 / 1$, the form drag for sphere \#1 reveals a local maximum at around $\beta=20$. At $\beta>20$ for $d_{f 1} / d_{f 2}=3 / 1$ or $\beta<>10$ for $d_{f 1} / d_{f 2}=1 / 3$, both drags level off and do not vary with $\beta$, revealing the "nonporous limit". Basically, the drag forces on sphere \#1 resemble qualitatively those for a single sphere moving in an unbound fluid, as the data presented in Fig. 6 (indicated as curves).

On the other hand, for sphere \#2, distinct dependence of drag forces on $\beta$ value is noticeable. For both cases shown in Fig. $7 \mathrm{~b}$ the skin drag is more significant than the form

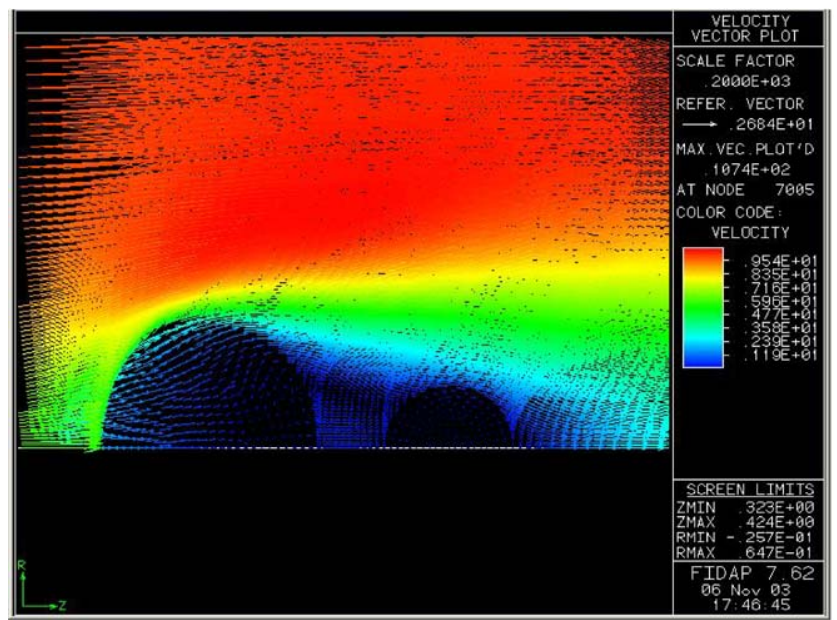

Fig. 8. The velocity fields for two spheres of $d_{f 1}=2 d_{f 2}$ and $\beta=10$. $S / d_{f 2}=2.5 . R e=40$.

drag. Moreover, the highly porous limits for sphere $\# 2$ are close to those for sphere $\# 1$ : $\beta<10$ for $d_{f 1} / d_{f 2}=3 / 1$ or $\beta<2$ for $d_{f 1} / d_{f 2}=1 / 3$. At $\beta>2$ and $d_{f 1} / d_{f 2}=3 / 1$, owing to the complicated interactions between sphere $\# 1$ and sphere \#2. Both form drag and skin drag show a complicated dependence on $\beta$ value, corresponding to the trend of total drag force noted in Fig. 6.

Fig. 8 presents the velocity field for the two spheres of different diameters $\left(d_{f 1} / d_{f 2}=2, S / d_{f 2}=2.5, \beta=10, R e=40\right)$. The wake flow by sphere \#1 is clearly observed in the figure, which affects the flow pattern around the sphere \#2. A wake exists at the regime after sphere \#1, which yields a flow over the front side of sphere \#2 toward the negative axis of centerline, and over the rear side of sphere toward the positive axis of centerline. Hence, the form drag is negative over the front side, and positive over the rear side of sphere $\# 2$, tend to pushing apart sphere \#2 along its centerline. Restated, the sphere \#2 is pulled apart at its both sides along the center-to-center axis. These two forces counterbalance each other, and yield a negative normal stress in the intermediate $\beta$ value. Meanwhile, the disturbed flow pattern around sphere \#2 also leads to negative shear stress over the same $\beta$ range.

Hence, the force field on sphere $\# 2$ at $d_{f 1} / d_{f 2}=3 / 1$ could be interpreted as follows. As $\beta$ increases up to 2.0, the drag force exerted on sphere \#2 increases, owing to the higher hydrodynamic resistance exerted with a less porous interior. At higher $\beta$ value the wake by sphere \#1 is quickly built up. Over $\beta=2-20$ the wake after sphere \#1 sufficiently shades the sphere $\# 2$ and reduce its drag. Beyond this $\beta$ value, since the shading effect has reached its maximum strength, the drag force monotonically increases at higher $\beta$ regime.

\subsection{Motion of porous spheres}

The $\Omega$ values for the two identical spheres moving in-line at creeping flow limit are the same (Fig. 5a). Hence, when two spheres are settled freely subjected to an external force 
field, such as to a gravitational field, a stable in-line settling is possible regardless of the porosity of their interior. On the other hand, at $R e=40$, the $\Omega$ value for sphere $\# 1$ at $\beta>1.5$ would be greater than the sphere \#2 (Fig. $5 b$ ). Hence, the two spheres would have no chance to settle stably in-line. Nonetheless, at $\beta<1.5$, this constraint is released and a stable in-line setting is again achievable. At $\beta>20$, the $\Omega$ values do not change with $\beta$ any more, corresponding to the "nonporous limit". Over the range $2<\beta<20$, the drag force on spheres is sensitive to the floc size and permeability, which are not easily quantitatively evaluated in practice. In applications involving wastewater flocs the corresponding $\beta$ values range $1.5-5.0$ for the original activated sludge flocs (Wu et al., 1998), and 5.0-20 for polyelectrolyte flocculated sludge flocs (Wu et al., 2000a,b). Restated, the $\beta$ values of real world flocs locate at the regime where the floc permeability and size considerably affect the drag force.

The flocs collected from the same suspension normally have a similar $\beta$ value (Wu et al., 2003). Restated, the permeability of a sphere is proportional to the square of its size. Sludge flocs are fractal-like objects whose interior compactness is characterized by fractal dimension, DF $(\leqslant 3)(\mathrm{Wu}$ et al., 2002b). Consider initially two spheres of different diameters moving in-line at the same velocity $(R e=40)$. The ratio of buoyant weights for the two spheres is proportional to $\left(d_{f 1} / d_{f 2}\right)^{\mathrm{DF}}$, and is equal to three for impermeable or homogeneously porous spheres. At $\left(d_{f 1} / d_{f 2}\right)=1 / 3$, the sphere $\# 1$ has a buoyant weight $1 / 27$ of that of sphere $\# 2$ if $D F=3$. The corresponding total drag forces at $\beta=100$ are 0.017 and 0.092 dynes, respectively (Fig. 6). Therefore, the large sphere \#2 will move faster than, and finally catch up sphere $\# 1$, during in-line settling. This observation corresponds to the so-called "sweeping coagulation". At $\left(d_{f 1} / d_{f 2}\right)=3$, the sphere \#1 has a buoyant weight 27 times of that of sphere $\# 2$ if $\mathrm{DF}=3$. The corresponding total drag forces at $\beta=100$ are 0.071 and 0.0013 dynes, respectively (Fig. 6). Hence, the smaller sphere \#2 will move faster than, and finally hit sphere \#1 during in-line settling. This occurrence also yield coagulation if the two spheres were bound together once collided.

\section{Conclusions}

This paper numerically evaluates the hydrodynamic drag force exerted on two highly porous spheres moving steadily along their centerline through a quiescent Newtonian fluid over a Reynolds number ranging from 0.1 to 40 . The governing equations are solved by the computational fluid dynamics software, FIDAP 7.6, from which the corresponding hydrodynamic drag force exerted on the floc is subsequently obtained. For highly porous spheres moving in-line, the fluid could not only flow around, but also flow through the porous sphere. Owing to the easy advection flow through the floc interior, the boundary layer separation and the after-sphere wakes did not occur even the spheres were moving at an intermediate Reynolds number. The mutual interactions between highly porous spheres were considerably weak while at dimensionless diameter $(\beta)<2$, the effects of separation distance and the diameters of spheres had no effects on their drag forces.

At creeping flow limit, the drag forces exerted on both spheres were identical. At a higher Reynolds number the drag force on sphere \#1 was higher than sphere \#2, revealing the shading effects produced by sphere \#1 on sphere \#2. The incorporation of inertia force weakens the effects of sphere $\# 2$ on sphere $\# 1$, but enhances the effects of sphere $\# 1$ on sphere $\# 2$. At $\beta>20$, the spheres can be regarded nonporous. At $\beta<20$, the drag forces dropped. At $\beta<2$, the drag forces approached "no-spheres" limit. An increased size ratio of two spheres $\left(d_{f 1} / d_{f 2}\right)$ would increase the drag force on sphere \#1 and reduce that on sphere \#2. The drag force exerted on sphere \#2 at $d_{f 1}>d_{f 2}$ would first increase with $\beta$ up to around 2 , then drop to negative and reach a local minimum at around $\beta=10$. Finally, the drag force increases slightly again when $\beta$ further goes up. This occurrence is attributable to the following competition on sphere \#2 at increasing $\beta$ : the higher drag force yielded by more difficult advective flow through the interior of sphere $\# 2$, and the lower drag force by stronger wake flow produced by the denser sphere \#1.

Two identical spheres could move steadily along their centerline. At higher Reynolds number, the two spheres tended to move closer because of the incorporation of inertia force. For spheres of different diameters, the sphere \#2 would move faster than sphere \#1 regardless of their size ratio and $\beta$ value. This occurrence will lead to coagulation if the two spheres were bound together once collided.

\section{Notation}

A proportionality constant in Stokes law-like correlation, dimensionless

$C_{D} \quad$ drag coefficient, dimensionless

$d_{f} \quad$ diameter of sphere, $\mathrm{m}$

$d_{f 1} \quad$ diameter of sphere $\# 1, \mathrm{~m}$

$d_{f 2} \quad$ diameter of sphere $\# 2, \mathrm{~m}$

$D_{F} \quad$ fractal dimension, dimensionless

$\mathrm{Eu} \quad$ Euler number $\left(=P_{0} / \rho V^{2}\right)$, dimensionless

$F_{D} \quad$ hydrodynamic resistance of porous sphere, dyne

$F_{D 1} \quad$ hydrodynamic drag force on sphere \#1, dyne

$F_{D 2} \quad$ hydrodynamic drag force on sphere \#2, dyne

$F_{\text {form }} \quad$ form drag, dyne

$F_{S} \quad$ hydrodynamic resistance of nonporous sphere in unbound fluid, dyne

$F_{\text {skin }} \quad$ skin drag, dyne

$k$

$k_{1}$ permeability of porous sphere, $\mathrm{m}^{2}$ permeability of porous sphere $\# 1, \mathrm{~m}^{2}$ 
$k_{2} \quad$ permeability of porous sphere $\# 2, \mathrm{~m}^{2}$

$L \quad$ length of circular tube, $\mathrm{m}$

$p \quad$ pressure, $\mathrm{N} / \mathrm{m}^{2}$

$P_{0} \quad$ reference pressure, $\mathrm{Pa}$

$R \quad$ radius of circular tube, $\mathrm{m}$

$r \quad$ radial direction, $\mathrm{m}$

Re Reynolds number, dimensionless

$S \quad$ center-to-center distance, $\mathrm{m}$

$\vec{u}_{f} \quad$ fluid velocity, $\mathrm{m} / \mathrm{s}$

$\vec{u}_{f}^{*} \quad=\vec{u}_{f} / V$, dimensionless

$\vec{u}_{p} \quad$ fluid velocity within the porous sphere, $\mathrm{m} / \mathrm{s}$

$\vec{u}_{p}^{*} \quad=\vec{u}_{p} / V$, dimensionless

$V \quad$ bulk velocity of fluid, $\mathrm{m} / \mathrm{s}$

\section{Greek letters}

$\beta \quad$ dimensionless permeability of sphere, dimensionless

$\gamma \quad$ correction factor in Eq. (2), dimensionless

$\theta \quad$ spherical coordinate, dimensionless

$\mu \quad$ viscosity of fluid, $\mathrm{Pa} \mathrm{s}$

$\rho \quad$ density of fluid, $\mathrm{kg} / \mathrm{m}^{3}$

$\tau_{r \theta} \quad$ shear stress on sphere's surface, $\mathrm{Pa}$

$\phi \quad$ spherical coordinate, dimensionless

$\Omega \quad$ correction factor based on single sphere of size $d_{f 2}$, dimensionless

$\Omega_{0} \quad$ correction factor for nonporous sphere, dimensionless

\section{References}

Bird, R.B., Warren, E.S., Lightfoot, E.N., 2002. Transport Phenomena, 2nd Edition. Wiley, New York.

Chen, R.C., Lu, Y.N., 1998. The flow characteristics of an interactive particle at low Reynolds number. ASME Fluids Engineering Division Summer Meeting. Washington, DC, June.

Chen, R.C., Wu, J.L., 2000. The flow characteristics between interactive spheres. Chemical Engineering Science 55, 1143-1158.

Chu, C.P., Feng, W.H., Tsai, I.H., Lee, D.J., 1997. Uni-directional freezing of waste activated sludge: the presence of sodium chloride. Environmental Science and Technology 31, 1512-1517.

Chung, H.Y., Ju, S.P., Lee, D.J., 2003. Hydrodynamic drag force exerted on waste activated sludge floc. Journal of Colloid and Interface Science, 263(2), 498-505.

Clift, R., Grace, J.R., Weber, M.E., 1978. Bubbles, Drops and Particles. Academic Press, New York.

Denn, M.M., 1980. Process Fluid Mechanics. Prentice-Hall Inc., Englewood Cliffs, NJ.

FIDAP, 1993. FIPOST Users Manual. Fluid Dynamics Int., Inc., Illinois, pp. $56-1$.

Happel, J., Brenner, H., 1983. Low Reynolds Number Hydrodynamics. Academic Press, New York.

Hung, W.T., Chang, I.L., Chen, G.W., Lee, D.J., 1996. Uni-directional freezing of waste activated sludge: the effects of freezing speed. Environmental Science and Technology 30, 2391-2396.
Lee, D.J., Hsu, Y.H., 1994. Fast freeze/thaw treatment on activated sludge: floc structure and sludge dewaterability. Environmental Science and Technology 28, 1444-1449.

Lee, D.J., Chen, G.W., Liao, Y.C., Hsieh, C.C., 1996. Use of free-settling test to estimate activated sludge flocs density. Water Research 30 , $541-550$.

Lee, K.C., 1979. Aerodynamic interaction between two spheres at Reynolds numbers around $10^{4}$. Aeroautical Quarterly 30, 371-385.

Liang, S.C., Hong, T., Fan, L.S., 1996. Effects of particle arrangements on the drag force of a particle in the intermediate flow regime. International Journal of Multiphase Flow 22, 285-306.

Matsumoto, K., Suganuma, A., 1977. Settling velocity of a permeable model floc. Chemical Engineering Science 32, 445-447.

Mitani, T., Unno, H., Akekata, T., 1983. A structure model for activated sludge flocs. Japan Water Research 6, 65-79.

Namer, J., Ganczarczyk, J., 1993. Settling properties of digested sludge particle aggregates. Water Research 27, 1285-1294.

Neale, G., Epstein, N., Nader, W., 1973. Creeping flow relative to permeable spheres. Chemical Engineering Science 28, 1865-1874.

Stimson, M., Jeffery, G.B., 1926. The motion of two spheres in a viscous fluid. Proceedings of Royal Society A111, 110-116.

Tambo, N., Watanabe, Y., 1979. Physical characteristics of flocs-I. The floc density function and aluminum floc. Water Research 13, 409-419.

Tsou, G.W., Wu, R.M., Yen, P.S., Lee, D.J., Peng, X.F., 2002. Advective flow and floc permeability. Journal of Colloid and Interface Science 250, 400-408.

Tsuji, Y., Morikawa, Y., Terashima, K., 1982. Fluid-dynamic interaction between two spheres. International Journal of Multiphase Flow 8, $71-82$.

Tsuji, Y., Morikawa, Y., Fujiwara, Y., 1985. Pipe flow with solid particles fixed in space. International Journal of Multiphase Flow 11, 177-188.

Wu, R.M., Lee, D.J., 1998a. Hydrodynamic drag force exerted on a moving floc and its implication to free-settling tests. Water Research 32, 760-768.

Wu, R.M., Lee, D.J., 1998b. Hydrodynamic drag force exerted on a highly porous sphere moving towards an impermeable plate. Chemical Engineering Science 53, 3571-3578.

Wu, R.M., Lee, D.J., 1999. Highly porous sphere moving through centerline of circular tube filled with Newtonian fluid. Chemical Engineering Science 54, 5717-5724.

Wu, R.M., Lee, D.J., 2001. Hydrodynamic drag on non-spherical floc and free-settling test. Water Research 35, 3226-3234.

Wu, R.M., Feng, W.H., Tsai, I.H., Lee, D.J., 1998. An estimate of waste activated sludge floc permeability: a novel hydrodynamic approach. Water Environment Research 70, 1258-1264.

Wu, R.M., Tsou, G.W., Lee, D.J., 2000a. Estimate of sludge floc permeability. Chemical Engineering Journal 80, 37-42.

Wu, R.M., Tsou, G.W., Lee, D.J., 2000b. Estimation of the interior permeability of polymer-flocculated sludge flocs. Advances in Environmental Research 4, 163-167.

Wu, R.M., Lee, D.J., Waite, T.D., Guan, J., 2002a. Multi-level structure of sludge flocs. Journal of Colloid and Interface Science 252, 383-392.

Wu, R.M., Tsou, G.W., Yen, P.S., Lee, D.J., Peng, X.F., 2002b. Tracking advective flow through floc interior. Journal of Chemical Engineering of Japan 35, 540-546.

Wu, R.M., Lee, D.J., He, P.J., 2003. Estimation of floc permeability and porosity. Journal of Chinese Institute of Chemical Engineers 34, $275-280$.

Zhu, C., Liang, S.C., Fan, L.S., 1994. Particle wake effects on the drag force of an interactive particle. International Journal of Multiphase Flow 20, 117-129. 